Topological Methods in Nonlinear Analysis

Volume 46, No. 2, 2015, 1029-1066

DOI: 10.12775 /TMNA.2015.076

(C) 2015 Juliusz Schauder Centre for Nonlinear Studies

Nicolaus Copernicus University

\title{
CONTRACTIVE FUNCTION SYSTEMS, THEIR ATTRACTORS AND METRIZATION
}

\author{
Taras Banakh — WiesŁaW Kubiś — Natalia Novosad \\ Magdalena NowAK — Filip Strobin
}

\begin{abstract}
In this paper we study the Hutchinson-Barnsley theory of fractals in the setting of multimetric spaces (which are sets endowed with point separating families of pseudometrics) and in the setting of topological spaces. We find natural connections between these two approaches.
\end{abstract}

\section{Introduction}

In this paper we consider topological and contracting properties of iterated function systems, well-known in the Theory of Fractals. By an Iterated Function System on a topological space $X$ we understand a dynamical system on the hyperspace $\mathcal{K}(X)$ of $X$, generated by a finite family $\mathcal{F}$ of continuous self-maps of $X$. In this case the finite family $\mathcal{F}$ will be called a function system on $X$.

By $\mathcal{K}(X)$ we denote the space of non-empty compact subsets of $X$ endowed with the Vietoris topology. This topology is generated by the subbase consisting

2010 Mathematics Subject Classification. Primary: 28A80; Secondary: 37C25, 37C70.

Key words and phrases. Fractal, attractor, iterated function system, contracting function system, fixed point.

Research of Wiesław Kubiś has been supported by GAČR grant P201 14 07880S.

Research of Magdalena Nowak has been partially supported by National Science Centre grant DEC-2012/07/N/ST1/03551.

Research of Filip Strobin has been partially supported by National Science Centre grant No. 2013/08/S/ST1/00541.

Research of Taras Banakh has been partially supported by National Science Centre grant No. 2012/07/D/ST1/02087. 
of the sets $\{K \in \mathcal{K}(X): K \cap U \neq \emptyset\}$ and $\{K \in \mathcal{K}(X): K \subset U\}$, where $U$ runs over open sets in $X$. If the topology of the space $X$ is generated by a metric $d$, then the Vietoris topology on $\mathcal{K}(X)$ is generated by the Hausdorff metric

$$
d_{H}(A, B)=\max \left\{\max _{a \in A} \min _{b \in B} d(a, b), \max _{b \in B} \min _{a \in A} d(a, b)\right\} .
$$

By $X^{X}$ we denote the semigroup of all self-maps of $X$, endowed with the operation of composition. The identity transformation $\operatorname{id}_{X}$ of $X$ is the (twosided) unit in the semigroup $X^{X}$, so $X^{X}$ is a monoid. For two subsets $\mathcal{A}, \mathcal{B} \subset X^{X}$ let $\mathcal{A} \circ \mathcal{B}=\{\alpha \circ \beta: \alpha \in \mathcal{A}, \beta \in \mathcal{B}\}$. Each subset $\mathcal{F} \subset X^{X}$ generates the submonoid $\mathcal{F}^{<\omega}=\bigcup_{n \in \omega} \mathcal{F}^{n}$ of $X^{X}$ where $\mathcal{F}^{0}=\left\{\mathrm{id}_{X}\right\}$ and $\mathcal{F}^{n+1}=\mathcal{F} \circ \mathcal{F}^{n}$ for $n \in \omega$. For every $k \in \omega$ the subset $\mathcal{F}^{\geq k}=\bigcup_{n \geq k} \mathcal{F}^{n}$ is a subsemigroup of $X^{X}$. For each subsets $\mathcal{F} \subset X^{X}$ and $A \subset X$ let $\mathcal{F}(A)=\bigcup_{f \in \mathcal{F}} f(A) \subset X$.

Any function system $\mathcal{F} \subset X^{X}$ (i.e. a finite family of continuous self-maps) on a topological space $X$ induces a continuous map $\mathcal{F}: \mathcal{K}(X) \rightarrow \mathcal{K}(X)$ assigning to each compact set $K \in \mathcal{K}(X)$ the compact set $\mathcal{F}(K)=\bigcup_{f \in \mathcal{F}} f(K)$ of $X$.

A non-empty compact set $A_{\mathcal{F}} \in \mathcal{K}(X)$ is called an attractor of a function system $\mathcal{F} \subset X^{X}$ if $\mathcal{F}\left(A_{\mathcal{F}}\right)=A_{\mathcal{F}}$ and for every compact set $K \in \mathcal{K}(X)$ the sequence $\left(\mathcal{F}^{n}(K)\right)_{n=1}^{\infty}$ converges to $A_{\mathcal{F}}$ in the Vietoris topology on $\mathcal{K}(X)$. In a Hausdorff topological space $X$ a finite system $\mathcal{F} \subset X^{X}$ of continuous selfmaps has at most one attractor.

Observe that a function system $\mathcal{F}$ on a topological space $X$ has an attractor if and only if the map $\mathcal{F}: \mathcal{K}(X) \rightarrow \mathcal{K}(X)$ has an attracting fixed point. We shall say that a self-map $f: X \rightarrow X$ of a topological space $X$ has an attracting fixed point if there is a point $x_{\infty} \in X$ such that $f\left(x_{\infty}\right)=x_{\infty}$ and for every point $x \in X$ the sequence $\left(f^{n}(x)\right)_{n=1}^{\infty}$ converges to $x_{\infty}$. This observation makes possible to apply results of Fixed Point Theory to finding attractors of function systems. In fact, the theory of functions systems with attractors has been deeply studied for the last thirty years - the great impact on this field had the papers [12] of Hutchinson and [3] of Barnsley (who applied the Banach Fixed Point Theorem), and [11] of Hata (who applied some other fixed point theorems). Note that the approach exploiting fixed point theorems is not the only one-before Theorem 1.1 we recall another one related to symbolic dynamics. In fact, this approach was the main one in the mentioned paper of Hutchinson, and also we will exploit it many times in this paper.

We are interested in detecting function systems possessing an attractor. A necessary condition for a function system $\mathcal{F}$ to have an attractor is the compact dominacy. We shall say that a function system $\mathcal{F}$ on a topological space $X$ is compact-dominating if each compact set $C \subset X$ is contained in a compact 